\title{
Das JVL geht in seinen 10. Jahrgang
}

\author{
Marion Rukavina
}

Published online: 21 February 2015

(C) Bundesamt für Verbraucherschutz und Lebensmittelsicherheit (BVL) 2015

„Wissenschaftlichen Erkenntnissen aus dem Verbraucherschutz eine Plattform geben." Dieses Versprechen gab der ehemalige Präsident des Bundesamts für Verbraucherschutz und Lebensmittelsicherheit und Herausgeber des JVL, Dr. Christian Grugel, in seinem Editorial der allerersten Ausgabe. Das war im Februar 2006. Seitdem hat sich vieles getan und verändert. Nicht nur, dass die Redaktion des JVL seit Kurzem mit Frau Dr. Birgit Schönig und mir erweitert wurde. Aufgrund der Digitalisierung hat sich auch das Verhalten bei der Nutzung von Fachinformationen grundlegend verändert. Unsere Leser nutzen und bevorzugen zunehmend das digitale Angebot des JVL. Interaktivität, Multimedialität und mobile Angebote werden relevanter. Diesen Herausforderungen müssen wir uns aktiv stellen. Neben den formalen und praktischen Veränderungen, die nicht zuletzt auch der Digitalisierung einer ganzen Branche zugrunde liegen, hat sich das JVL auch an politische Geschehnisse anpassen müssen. Im Zuge der Regierungsneubildung im Jahre 2013 wurden der rechtliche und wirtschaftliche Verbraucherschutz dem jetzigen Bundesministerium der Justiz und für Verbraucherschutz zugeteilt. Das Bundesministerium für Ernährung und Landwirtschaft (BMEL) konzentriert sich beim Thema Verbraucherschutz nun auf die Sicherheit von Lebensmitteln und

\footnotetext{
M. Rukavina ( $\square)$

Journal für Verbraucherschutz und Lebensmittelsicherheit (JVL), Bundesamt für Verbraucherschutz und Lebensmittelsicherheit (BVL), Mauerstraße 39-42, 10117 Berlin, Germany

e-mail: jvl@bvl.bund.de
}

Bedarfsgegenständen. Diese Änderung spiegelt sich auch in den Inhalten des JVL wider.

Ein Thema, das derzeit auch den gesundheitlichen Verbraucherschutz beschäftigt, ist die anstehende Transatlantische Handels- und Investitionspartnerschaft (TTIP) mit den USA. Viele Verbraucher sind diesbezüglich stark verunsichert und dadurch teilweise komplett gegen das Abkommen, vor allem weil sie schlechtere Standards bei den Lebensmitteln-z. B. das berühmte Chlorhühnchen-fürchten. Während Verbraucherschützer grundsätzlich für den Freihandel sind, warnen sie vor dem Verlust europäischer Standards. So forderte der Leiter der Verbraucherzentrale Bundesverband (vzbv), Klaus Müller, im WISO-Magazin des ZDF: „Die Standards dürfen nicht ausgehöhlt und geopfert werden.“ Eine Möglichkeit, TTIP zu nutzen, wäre beispielsweise, die Standards für den Verbraucherschutz in den USA und in der EU anzugleichen, etwa durch gleiche Regeln für den Rückruf von Produkten oder die europäische Kennzeichnungspflicht für Nahrungsmittel auch in den USA.

\section{Verbraucherschutz, Trends und Innovationen}

Das TTIP steht auch im Zusammenhang mit dem sogenannten „Convenience Food“. Derzeit werden zwei Drittel der Lebensmittel industriell verarbeitetmit steigender Tendenz. So passen Fertiggerichte immer besser zum heutigen Zeitgeist des modernen, globalisierten Leistungsmenschen: Für Planung und Zubereitung von Mahlzeiten bleiben immer weniger Zeit und Energie. Welche Auswirkungen haben diese Trends beispielsweise auf die Lebensmittelsicherheit 
und Verpackungen im Haushalt? Durch welche Maßnahmen können Lebensmittel am besten geschützt und ihre Sicherheit gewährleistet werden? Welche Materialen und Designs stehen hierzu künftig zur Verfügung? In der Rubrik „Announcement and Reports“ der vorliegenden Ausgabe bieten wir Ihnen hierzu mögliche Antworten. Die Referenten des Hamburger Food Science Dialogs 2014 berichten darin vom zweiten Teil der jährlich stattfindenden Tagung, mit dem Schwerpunkt „Lebensmittelsicherheit und Verpackung“. Experten aus der Verpackungsindustrie, Lebensmittelüberwachung sowie aus Prüfinstituten und Hochschulen besprechen in ihren Beiträgen sowohl Sicherheit als auch Innovationen aus dem Bereich Lebensmittelverpackungen. Neben neuen Lösungen zur Minimierung von Migrationsphänomenen bei Papier- und Kartonverpackungen durch die Verwendung von BarriereSchichten oder Adsorptionsmaterialien, werden auch die vielseitigen aktuellen Anforderungen an flexible Lebensmittelverpackungen diskutiert.

Gleichzeitig haben Verbraucher immer höhere Erwartungen an die Lebensmittelqualität und legen großen Wert auf frische und gesunde Produkte. Sie interessieren sich zunehmend für deren Eigenschaften und Zusammensetzung. Als Information dient dem Konsumenten hierzu die Verkehrsbezeichnung auf Fertigverpackungen von Lebensmitteln, deren Angabe gesetzlich verpflichtend ist. Diese kurz gefasste Charakterisierung der Produktart auf den Verpackungen soll dem Verbraucher als eindeutiges Erkennungsmerkmal dienen und es ihm ermöglichen, verschiedene Erzeugnisse voneinander zu unterscheiden. Dabei kommt es immer wieder $\mathrm{zu}$ Konflikten zwischen Herstellern und Verbrauchervertretern, da manche Bezeichnungen auf den Lebensmitteln für den Nutzer irreführend sein können. Die Autoren R. Weinrich et al. haben anhand von drei Beispielprodukten innerhalb einer repräsentativen Verbraucherbefragung überprüft, ob das Begriffsverständnis der Konsumenten mit der tatsächlichen Zusammensetzung der Produkte übereinstimmt.

In der Rubrik „Review-Articles“ der vorliegenden Ausgabe finden Sie einen Beitrag zu einem Thema, das derzeit ebenfalls viele Gemüter bewegt: Der Einsatz von Glyphosat und das Ausmaß der Belastung bei Betreibern und Lebensmittel-Konsumenten. Als
Wirkstoff ist Glyphosat weltweit in vielen zugelassenen Pflanzenschutzmitteln enthalten; im Zuge der Anwendung können Glyphosat-Rückstände in Lebensmitteln gelangen. Laut einer Stellungnahme des Bundesinstituts für Risikobewertung (BfR) vom 6. März 2014 ist das Auftreten solcher Rückstände gesundheitlich unbedenklich-solange die gesetzlich festgelegten Höchstgehalte nicht überschritten werden. Ein Nachweis von Glyphosat im menschlichen Urin ist zu erwarten, da es hauptsächlich über die Nieren bzw. den Urin ausgeschieden wird. In ihrem Übersichtsartikel verglichen die Autoren L. Niemann et al. die Daten aus sieben Studien aus Europa und den USA und zogen daraus Rückschlüsse hinsichtlich des gesundheitlichen Risikos durch Glyphosat und seine Abbauprodukte. Lesen Sie mehr dazu in der entsprechenden Rubrik.

\section{Auf die nächsten 10 Jahrgänge}

Das JVL befindet sich in seinem zehnten Jahrgangwie geht es weiter? Von der ersten Ausgabe an vereinte das JVL begutachtete wissenschaftliche Artikel und behördliche Mitteilungen zum Verbraucherschutz. Dieses Format hat sich bewährt und wird auch weiterhin so fortbestehen. Im Jahre 2009 wurde die Zeitschrift zum ersten Mal beim „Institute for Scientific Information“ (ISI) mit einem Impact-Faktor gelistet und wir freuen uns, dass dieser Wert seither kontinuierlich gestiegen ist. An dieser Stelle möchten wir uns auch herzlich bei unseren Autoren und Gutachtern für die professionelle Arbeit bedanken!

Durch die fortschreitende Digitalisierung und Globalisierung kommen weitere Änderungen und Herausforderungen auf uns zu, die möglicherweise sowohl formale als auch inhaltliche Aspekte der Heftgestaltung betreffen werden. Für uns bedeutet dies, weiterhin dem Risikomanagement und dem Verbraucherschutz innerhalb des Zuständigkeitsbereichs des Bundesamtes für Verbraucherschutz und Lebensmittelsicherheit (BVL) eine lebendige Plattform zu bieten, die sowohl die behördliche als auch wissenschaftliche Kommunikation in sich vereint. Mit und trotz aller Veränderungen ist diese grundlegende Zielsetzung des JVL seit der ersten Ausgabe unverändert geblieben. 